\title{
Probing a Color Glass Condensate in High Energy Heavy Ion Collisions
}

\author{
A. Krasnitz ${ }^{a}$ Y. Nara ${ }^{b}$, and R. Venugopalan ${ }^{b}$ \\ ${ }^{a}$ CENTRA, Universidade do Algarve, Campus de Gambelas, P-8000 Faro, Portugal, \\ ${ }^{b}$ RIKEN-BNL Research Center, Brookhaven National Laboratory, Upton, NY-11973, USA
}

Received on 30 October, 2002

\begin{abstract}
At very high energies, the partons in the nuclear wavefunction form a color glass condensate. Since the occupation number of partons in the color glass condensate is large, classical methods can be used to compute multi-particle production in the initial instants of a high energy heavy ion collision. Non-perturbative expressions are derived relating the distributions of produced partons to those of wee partons in the wavefunctions of the colliding nuclei. The time evolution of components of the stress-energy tensor is studied and the impact parameter dependence of elliptic flow is extracted. We discuss the space-time picture that emerges and interpret the RHIC data within this framework.
\end{abstract}

\section{Introduction}

At the Relativistic Heavy Ion Collider (RHIC), beams of Gold ions collide at center of mass energies of $\sqrt{s_{N N}}=200$ $\mathrm{GeV} /$ nucleon. The goal is to create briefly an equilibrated state of quarks and gluons called the quark gluon plasma and to study its statistical properties [1], in particular its change of phase to hadronic matter. It was understood very early on that the likelihood of creating this novel state of matter depended crucially on the initial conditions for the collision $[2,3,4,5]$. There are several time scales in the problem and the appropriate values of these are determined by the initial conditions.

It was also understood very early on that the initial conditions for high energy collions are determined by the "wee partons" (partons that carry a very small fraction $x$ of the nuclear momentum) in the wavefunctions of the colliding nuclei [2]. This is because, in the language of quantum mechanics, small $x$ refers to Fock components of the nuclear wavefunction that contain a large number of partons (mostly gluons) [6]. In a nuclear collision, these virtual excitations of the vacuum go on-shell and are therefore responsible for multi-particle production. Thus an understanding of small $x$ physics is essential to any formulation of a theory of heavy ion collisions.

The problem of initial conditions is a difficult one because the behavior of the wee partons is mysterious and defies our naive intuition. For instance, wee partons are long wavelength excitations of the vacuum but they last for very short times ${ }^{1}$. The large coherence length of the excitations is also why a probabilistic picture of multi-particle production (as implemented, for instance, in parton cascade mod- els) must fail at high energies.

A traditional view is that the physics of wee partons is intrinsically non-perturbative-for example, multi-particle production is believed to be determined by non-perturbative excitations, called Pomerons, with vacuum quantum numbers $[8,9]$. It is believed that Pomerons could be constructed in perturbation theory (the BFKL Pomeron [7]) but the status of that approach is at present unclear [10]. An alternative, increasingly popular, viewpoint is that small $x$ physics is weak coupling physics. This approach is motivated by the idea of saturation [11], namely, that at small $x$ the density of partons could be sufficiently large that recombination and screening effects are significant enough to halt the growth of parton distributions ${ }^{2}$. The large parton density provides a semi-hard scale-the saturation scale $\Lambda_{s}$ - that controls the running of the QCD coupling constant- thereby making weak coupling methods feasible. Another consequence of this approach is that small $x$ physics is classical because the occupation number of partons is $\sim 1 / \alpha_{S}\left(\Lambda_{s}\right)>>1$ [13].

Both of these ideas, the weak coupling due to high parton densities and the applicability of classical methods can be cast in the framework of an effective field theory (EFT) [13] which treats partons at large $x$ as static sources of color charges for the to partons at small $x$. For a large nucleus, from the central limit theorem, these sources of color charge are Gaussian weights, $P[\rho]=$ $\exp \left(-\int d^{2} x_{t} \frac{1}{\Lambda_{s}^{2}} \operatorname{Tr}\left(\rho^{2}\right)\right)$ where the color charge charge density squared per unit area, $\Lambda_{s}^{2}$, interestingly, is the saturation scale we mentioned previously. The classical theory for a single nucleus is solvable analytically and the distributions of partons computed [14]. What is ad hoc in the

\footnotetext{
${ }^{1}$ This is why, from the uncertainity principle, one needs very high energies to probe these excitations.

${ }^{2}$ For a discussion of an alternative "final state" saturation scenario, see Refs. [12]
} 
classical picture is the separation between static sources and dynamical fields. Remarkably, a Wilsonian renormalization group procedure has been developed which quantifies this separation of scales in $x$ in a systematic way JIMWLK. The structure of the classical field is preserved under evolution; it is the weight function $P[\rho]$ in the effective action that obeys a renormalization group equation. The saturation scale (whose validity extends beyond the Gaussian model) now acquires energy dependence-it is a function $\Lambda_{s}(x)$ of $x$. The analogy of the EFT to spin glasses, and the high occupation number of fields with the momenta peaked at $\Lambda_{s}$ suggests that matter in this state is a Color Glass Condensate (CGC) [13, 16, 17].

Our focus in this talk is on applying the Color Glas Condensate to nuclear collisions. In the following section, we will outline the classical formalism for nuclear collisions. In section 3, we will apply this formalism to compute energy and number distributions of the gluons produced in a heavy ion collision. To study non-central collisions, we will have to consider collisions of non-identical nuclei and that will require we impose stringent constraints on color neutrality at the nucleon level. These improvements allow us to discuss elliptic and radial flow as well. In the final section we will discuss an interpretation of the RHIC data and shall conclude with brief outline of open problems and potential solutions in the classical approach.

\section{Classical formalism for nuclear collisions}

The classical EFT was first applied to the study of collisions of large nuclei by Kovner, McLerran and Weigert [18]. The model, as applied to nuclear collisions, may be summarized as follows. The colliding nuclei are idealized to travel along the light cone The high- $x$ and the low- $x$ modes in the nuclei are treated separately. The former corresponds to valence quarks and hard sea partons and are considered recoilless sources of color charge. Each of the large Lorentzcontracted nuclei (for simplicity, we will consider only collisions of identical nuclei) now has a Gaussian distribution of their color charge density $\rho_{1,2}$ in the transverse plane. The variance $\Lambda_{s}$ of the color charge distribution is the only dimensionful parameter of the model, apart from the linear size of the nucleus. For central impact parameters, $\Lambda_{s}$ can be estimated in terms of single-nucleon structure functions [?]. It is assumed, in addition, that the nucleus is infinitely thin in the longitudinal direction. Under this simplifying assumption, the resulting gauge fields are explicitly boost-invariant.

The small $x$ fields are then described by the classical Yang-Mills equations $D_{\mu} F_{\mu \nu}=J_{\nu}$ with the random sources on the two light cones: $J_{\nu}=$ $\sum_{1,2} \delta_{\nu, \pm} \delta\left(x_{\mp}\right) \rho_{1,2}\left(r_{t}\right)$. The two signs correspond to two possible directions of motion along the beam axis $z$. As shown by Kovner, McLerran and Weigert (KMW) [18], low- $x$ fields in the central region of the collision obey sourceless Yang-Mills equations (this region is in the forward light cone of both nuclei) with the initial conditions in the $A_{\tau}=0$ gauge given by $A^{i}=A_{1}^{i}+A_{2}^{i}$ and $A^{ \pm}= \pm \frac{i g}{2} x^{ \pm}\left[A_{1}^{i}, A_{2}^{i}\right]$. Here the pure gauge fields $A_{1,2}^{i}$ are solutions of (??) for each of the two nuclei in the absence of the other nucleus.

In order to obtain the resulting gluon field configuration at late proper times, one needs to solve the YM-equations with the above mentioned initial conditions. Since the latter depends on the random color source, averages over different realizations of the color sources must be performed. KMW showed that in perturbation theory the gluon number distribution by transverse momentum (per unit rapidity) suffers from an infrared divergence and argued that the distribution must have the form $n_{k_{\perp}} \propto \frac{1}{\alpha_{s}}\left(\frac{\Lambda_{s}}{k_{\perp}}\right)^{4} \ln \left(\frac{k_{\perp}}{\Lambda_{s}}\right)$ for $k_{\perp} \gg \Lambda_{s}$. The log term clearly indicates that the perturbative description breaks down for $k_{\perp} \sim \Lambda_{s}$.

A reliable way to go beyond perturbation theory is to reformulate the EFT on a lattice by discretizing the transverse plane. The resulting lattice theory can then be solved numerically to all orders in the color charge densities $\rho_{1}$ and $\rho_{2}$. The lattice Hamiltonian is formulated in $A^{\tau}=0$ gauge. The real time gluodynamics of gauge fields can then be studied by solving Hamilton's equations on the lattice. We shall not dwell here on the details of the lattice formulation, which is described in detail in Ref. [20, 21]. We will first consider, for simplicity, collisions of uniform, cylindrical nuclei. Keeping in mind that $\Lambda_{s}$ and the linear size $L$ of the nucleus ${ }^{3}$ are the only physically interesting dimensional parameters of the model [16], we can write any dimensional quantity $q$ as $\Lambda_{s}^{d} f_{q}\left(\Lambda_{s} L\right)$, where $d$ is the dimension of $q$. All the non-trivial physical information is contained in the dimensionless function $f_{q}\left(\Lambda_{s} L\right)$. We can estimate the values of the product $\Lambda_{s} L$ which correspond to key collider experiments. Assuming Au-Au collisions, we take $L=11.6 \mathrm{fm}$ (for a square nucleus!) and estimate the saturation scale $\Lambda_{s}$ to $\sim 1.4 \mathrm{GeV}$ for RHIC and $\approx 2.2 \mathrm{GeV}$ for LHC [24].

Also, we have approximately $g=2$ for energies of interest. The rough estimate is then $\Lambda_{s} R \approx 45$ (for RHIC and $\Lambda_{s} R \approx 72$ for LHC. Since the gluon distribution in nuclei is not known to great precision, there is a considerable systematic uncertainty in these estimates. We find that, this uncertainity notwithstanding, the dependence of our results on $\Lambda_{s} R$ is rather weak in the broad regime of interest.

The assumption of uniform, cylindrical nuclei is clearly not realistic since nuclear matter is not uniformly distributed in a nucleus. Therefore, in general, we expect the saturation scale to vary from point to point in the transverse plane, namely, $\Lambda_{s} \equiv \Lambda_{s}\left(x_{t}\right)$. Furthermore, since the initial conditions for a heavy ion collision at a fixed energy can be varied by varying the centrality of the collisions, it will be important to extend our previous considerations to collisions of finite nuclei. The most important consideration in this case is that the color charge of the quark and gluon fields in a nucleus remain confined inside its radius. That this is the case

\footnotetext{
${ }^{3} L$ is the length scale for a cylindrical nucleus; $L^{2}=\pi R^{2}$ where $R$ is the radius of the nucleus.
} 
is not a natural consequence of our picture and additional constraints have to be imposed on the color charge distributions of the sources to ensure that the classical gluon fields do not "leak" outside the nucleus [24]. These constraints, termed Color Neutral I and Color Neutral II in the following, respectively require that the monopole and dipole components of the source color charge density be set to zero. The results from these color neutrality constraints will be contrasted below with those from the global color charge constraint (namely, only the color charge density integrated over the entire nucleus is set to zero) imposed in our earlier studies.

\section{Energy and Number distributions of produced gluons}

The classical formalism has been applied to study classical gluon production arising from the "melting" of the Color Glass Condensate. The energy and number distributions were initially computed numerically for central collisions of uniform cylindrical nuclei and the dependence of these quantities on $\Lambda_{s}$ was determined [21, 22]. The initial simulations were performed for an $\mathrm{SU}(2)$ gauge theory $[21,22]$. These simulations were extended to an SU(3) gauge theory in Ref. [23]. Recently, these distributions have been obtained for an SU(3) gauge theory for finite nuclei with realistic initial conditions [24].
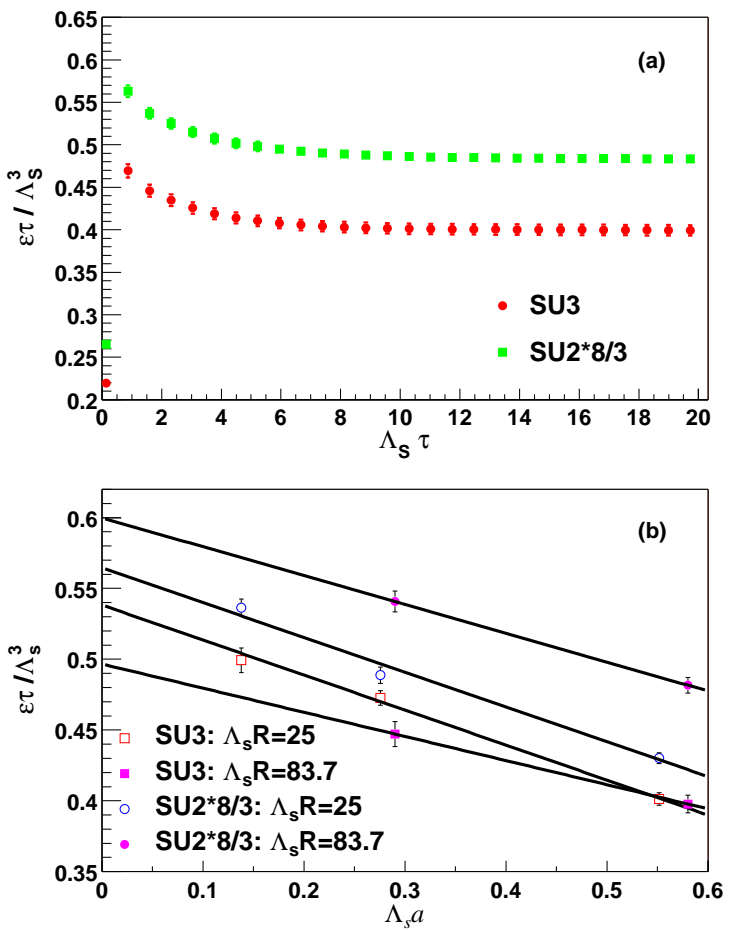

Figure 1. (a) $\varepsilon \tau / \Lambda_{s}^{3}$ as a function of $\tau \Lambda_{s}$ for $\Lambda_{s} R=83.7$. (b) $\varepsilon \tau / \Lambda_{s}^{3}$ as a function of $\Lambda_{s} a$ for $\Lambda_{s} R=83.7$ (squares) and 25(circles), where $a$ is the lattice spacing. Lines are fits of the form $a-b x$.
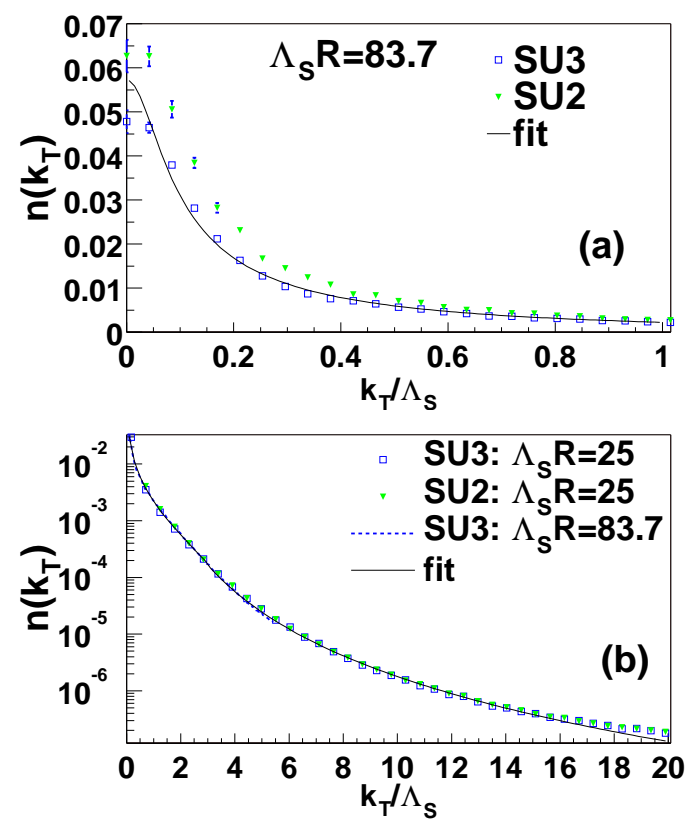

Figure 2. Transverse momentum distribution of gluons, normalized to the color degrees of freedom, $n\left(k_{T}\right)=\tilde{f}_{n} /\left(N_{c}^{2}-1\right)$ (see Eq. (2)) as a function of $\Lambda_{S} R$ for SU(3) (squares) and SU(2) (diamonds). Solid lines correspond to the fit in Eq.(3).

For the transverse energy of gluons, we obtain the relation

$$
\left.\frac{1}{\pi R^{2}} \frac{d E_{T}}{d \eta}\right|_{\eta=0}=\frac{1}{g^{2}} f_{E}\left(\Lambda_{s} R\right) \Lambda_{s}^{3},
$$

The function $f_{E}$ is determined non-perturbatively as follows. In Fig. 1(a), we plot the Hamiltonian density, for a particular fixed value of $\Lambda_{s} R=83.7$ (on a $512 \times 512$ lattice) in dimensionless units as a function of the proper time in dimensionless units. We note that in the SU(3) case, as in $\mathrm{SU}(2), \varepsilon \tau$ converges very rapidly to a constant value. The form of $\varepsilon \tau$ is well parametrized by the functional form $\varepsilon \tau=\alpha+\beta \exp (-\gamma \tau)$. Here $d E_{T} / d \eta / \pi R^{2}=\alpha$ has the proper interpretation of being the energy density of produced gluons, while $\tau_{D}=1 / \gamma / \Lambda_{s}$ is the "formation time" of the produced glue.

In Figure 1(b), the convergence of $\alpha$ to the continuum limit is shown as a function of the lattice spacing in dimensionless units for two values of $\Lambda_{s} R$. In Ref. [21], this convergence to the continuum limit was studied extensively for very large lattices (up to $1024 \times 1024$ sites) and shown to be linear. The trend is the same for the SU(3) results. Thus, despite being further from the continuum limit for $\mathrm{SU}(3)$ (due to the significant increase in computer time), a linear extrapolation is justified. We can therefore extract the continuum value for $\alpha$. We find $f_{E}(25)=0.537$ and $f_{E}(83.7)=0.497$. The RHIC value likely lies in this range of $\Lambda_{s} R$. The formation time $\tau_{D}=1 / \gamma / \Lambda_{s}$ is essentially the same for $\mathrm{SU}(2)$-for $\Lambda_{s} R=83.7, \gamma=0.362 \pm 0.023$. As discussed in Ref. [21], it is $\sim 0.3 \mathrm{fm}$ for RHIC and $\sim 0.13$ fm for LHC (taking $\Lambda_{s}=2 \mathrm{GeV}$ and $4 \mathrm{GeV}$ respectively).

We now combine our expression in Eq. (1) with our non-perturbative expression for the formation time to obtain a non-perturbative formula for the initial energy density, 
$\varepsilon=\frac{0.17}{g^{2}} \Lambda_{s}^{4}$. This formula gives a rough estimate of the initial energy density, at a formation time of $\tau_{D}=1 / \bar{\gamma} / \Lambda_{s} R$ where we have taken the average value of the slowly varying function $\gamma$ to be $\bar{\gamma}=0.34$.

To determine the gluon number per unit rapidity, we first compute the gluon transverse momentum distributions. The procedure followed is identical to that described in Ref. [22] -we compute the number distribution in Coulomb gauge, $\nabla_{\perp} \cdot A_{\perp}=0$. In Fig. 2(a), we plot the normalized gluon transverse momentum distributions versus $k_{T} / \Lambda_{s}$ with the value $\Lambda_{s} R=83.7$, together with SU(2) result. Clearly, we see that the normalized result for SU(3) is suppressed rel- ative to the $\mathrm{SU}(2)$ result in the low momentum region. In Fig. 2(b), we plot the same quantity over a wider range in $k_{T} / \Lambda_{s}$ for two values of $\Lambda_{s} R$. At large transverse momentum, we see that the distributions scale exactly as $N_{c}^{2}-1$, the number of color degrees of freedom. This is as expected since at large transverse momentum, the modes are nearly those of non-interacting harmonic oscillators. At smaller momenta, the suppression is due to non-linearities, whose effects, we have confirmed, are greater for larger values of the effective coupling $\Lambda_{s} R$.

The SU(3) gluon momentum distribution can be fitted by the following function,

where $\tilde{f}_{n}\left(k_{T} / \Lambda_{s}\right)$ is

$$
\frac{1}{\pi R^{2}} \frac{d N}{d \eta d^{2} k_{T}}=\frac{1}{g^{2}} \tilde{f}_{n}\left(k_{T} / \Lambda_{s}\right)
$$

$$
\tilde{f}_{n}= \begin{cases}a_{1}\left[\exp \left(\sqrt{k_{T}^{2}+m^{2}} / T_{\text {eff }}\right)-1\right]^{-1} & \left(k_{T} / \Lambda_{s} \leq 3\right) \\ a_{2} \Lambda_{s}^{4} \log \left(4 \pi k_{T} / \Lambda_{s}\right) k_{T}^{-4} & \left(k_{T} / \Lambda_{s}>3\right)\end{cases}
$$

with $a_{1}=0.0295, m=0.067 \Lambda_{s}, T_{\text {eff }}=0.93 \Lambda_{s}$, and $a_{2}=0.0343$. At low momenta, the functional form is approximately that of a Bose-Einstein distribution in two dimensions even though the underlying dynamics is that of classical fields. The functional form at high momentum is motivated by the lowest order perturbative calculations $[19,18,26]$.

Integrating our results over all momenta, we obtain for the gluon number per unit rapidity, the non-perturbative result, $\left.\frac{1}{\pi R^{2}} \frac{d N}{d \eta}\right|_{\eta=0}=\frac{1}{g^{2}} f_{N}\left(\Lambda_{s} R\right) \Lambda_{s}^{2}$. We find that $f_{N}(83.7)=0.3$. The results for a wide range of $\Lambda_{s} R$ vary on the order of $10 \%$ in the case of SU(2).

For realistic nuclei, these non-perturbative relations are less simple. One can parametrize our results for the gluon number with the more general relation

$$
\frac{d N_{g}}{d \eta}=f_{N}(b) \int d^{2} x_{T} \frac{\Lambda_{s}^{2}\left(b, x_{T}\right)}{g^{2}}
$$

where $\Lambda_{s}\left(b, x_{T}\right)$ is the local saturation scale defined to be $\Lambda_{s}^{2}\left(b, x_{T}\right)=C \cdot \tilde{\rho}\left(b, x_{T}\right) / 2$, where $\tilde{\rho}$ is the participant density at a particular position in the transverse plane, and $C$ is the color charge squared per nucleon. When $\Lambda_{s}\left(b, x_{T}\right)=$ constant, as for cylindrical uniform nuclei, one recovers the form of the expressions in Refs. [22, 23]. The color charge squared in the center of the nucleus is $\Lambda_{s 0}^{2}=$ $C \cdot \tilde{\rho}(0,0) / 2$, so $\Lambda_{s}^{2}\left(b, x_{T}\right)=\Lambda_{s 0}^{2} \tilde{\rho}\left(b, x_{T}\right) / \tilde{\rho}(0,0)$. One can then re-write the previous equation as

$$
\frac{d N_{g}}{d \eta}=\frac{f_{N}(b)}{g^{2}} \frac{\Lambda_{s 0}^{2}}{\rho_{0}} N_{\text {part }}(b)
$$

where $\rho_{0}=\tilde{\rho}(0,0)=4.321 \mathrm{fm}^{-2}$ and $N_{\text {part }}=$ $\int d^{2} x_{T} \tilde{\rho}\left(b, x_{T}\right)$.

In Tables I and II, we show the calculated SU(3) results for two values of the saturation scale in the center of the nucleus: $\Lambda_{s 0}=1.41$ and $\Lambda_{s 0}=2.32 \mathrm{GeV}$ respectively. In the tables, $b$ is an impact parameter (in units of fm) and $N_{\text {part }}$ is a number of participants at that impact parameter. The latter is calculated using a Woods-Saxon nuclear density profile. We list in the tables our results, as a function of impact parameter, for $g^{2} N_{g}$; the number of produced gluons and $g^{2} E_{g}$; the transverse energy of produced gluons in $\mathrm{GeV}$ multiplied by the value of the strong coupling constant squared $g^{2}$, evaluated (to one loop order) at the average value of the saturation scale (denoted in the tables as $Q_{s}(b)$ ) for that impact parameter.

Table I. $\Lambda_{s 0}=1.41 \mathrm{GeV}$. In the calculation, lattice size of $256 \times 256$ and nuclear radius of 64 in lattice units is used. All dimensionful scales are in $\mathrm{GeV}$ units unless otherwise stated.

\begin{tabular}{lllllll}
$b(\mathrm{fm})$ & $N_{\text {part }}$ & $g^{2} N_{g}$ & $g^{2} E_{g}$ & $\Lambda(b)$ & $Q_{s}(b)$ & $f_{N}(b)$ \\
\hline 0.000 & 377.89 & 1628.68 & 2725.40 & 1.1777 & 1.0836 & 0.3695 \\
3.150 & 321.35 & 1309.83 & 2170.08 & 1.1545 & 1.0563 & 0.3484 \\
4.725 & 263.33 & 1035.84 & 1663.88 & 1.1234 & 1.0197 & 0.3359 \\
6.300 & 199.11 & 760.95 & 1182.02 & 1.0741 & 0.9623 & 0.3249 \\
7.875 & 136.47 & 515.61 & 751.902 & 0.9993 & 0.8758 & 0.3152 \\
9.450 & 81.21 & 295.76 & 384.004 & 0.8876 & 0.7487 & 0.3004
\end{tabular}


Table II. $\Lambda_{s 0}=2.32 \mathrm{GeV}$. In the calculation, lattice size of $512 \times 512$ and nuclear radius of 128 in lattice units is used. All dimensionful scales are in $\mathrm{GeV}$ units unless otherwise stated.

\begin{tabular}{lllllll}
$b(\mathrm{fm})$ & $N_{\text {part }}$ & $g^{2} N_{g}$ & $g^{2} E_{g}$ & $\Lambda(b)$ & $Q_{s}(b)$ & $f_{N}(b)$ \\
\hline 0.000 & 377.89 & 3768.00 & 9198.736 & 1.9517 & 2.0355 & 0.2867 \\
3.150 & 321.35 & 3061.59 & 7492.084 & 1.9132 & 1.9866 & 0.2757 \\
6.300 & 199.11 & 1808.89 & 4183.888 & 1.7800 & 1.8185 & 0.2610 \\
7.875 & 136.47 & 1215.17 & 2636.300 & 1.6560 & 1.6636 & 0.2522 \\
8.367 & 118.17 & 1042.54 & 2243.692 & 1.6060 & 1.6017 & 0.2514 \\
9.450 & 81.21 & 699.95 & 1411.900 & 1.4708 & 1.4356 & 0.2411
\end{tabular}

\section{Elliptic Flow}

The azimuthal anisotropy in the transverse momentum distribution has been proposed as a sensitive probe of the hot and dense matter produced in ultra-relativistic heavy ion collisions [27]. A measure of the azimuthal anisotropy is the second Fourier coefficient of the azimuthal distribution, the elliptic flow parameter $v_{2}$. Its definition [28] is

$$
v_{2}=\langle\cos (2 \phi)\rangle=\left\langle\frac{p_{x}^{2}-p_{y}^{2}}{p_{x}^{2}+p_{y}^{2}}\right\rangle=\frac{\int_{-\pi}^{\pi} d \phi \cos (2 \phi) \int p_{T} d p_{T} \frac{d^{3} N}{d y p_{T} d p_{T} d \phi}}{\int_{-\pi}^{\pi} d \phi \int p_{T} d p_{T} \frac{d^{3} N}{d y p_{T} d p_{T} d \phi}}
$$

The first measurements of elliptic flow from RHIC, at center of mass energy $\sqrt{s_{N N}}$, have been reported recently [29]. Hydrodynamic model calculations provide good agreement, for large centralities, and for particular initial conditions and equations of state, with the measured centrality dependence of the data. The agreement at smaller centralities is less good, perhaps reflecting the breakdown of a hydrodynamic description in smaller systems. Hydrodynamic models are also in excellent agreement with the $p_{t}$ dependence of the unintegrated elliptic flow parameter $v_{2}\left(p_{t}\right)$ up to $1.5 \mathrm{GeV} / \mathrm{c}$ at mid-rapidity [30]. However, above 1.5 $\mathrm{GeV}$, the experimental distribution appears to saturate, while the hydrodynamic model distribution continues to rise. It has been argued recently that jet quenching might explain this saturated behavour of $v_{2}\left(p_{t}\right)$ [31]. We should also note here that hadronic transport model calculations underestimate the RHIC $v_{2}$ data[29, 32].

We will now apply the classical Yang-Mills approach to compute the elliptic flow generated in a nuclear collision. As previously, we assume boost invariance-the lattice Hamiltonian is the Kogut-Susskind Hamiltonian in 2+1-dimensions coupled to an adjoint scalar field in $A^{\tau}=0$ gauge [20]. In our earlier work, periodic boundary conditions were imposed to compute the space-time evolution of the gauge fields after the collision [21, 22, 23]. Since, as discussed previously, elliptic flow is a consequence of an initial spatial anisotropy, periodic boundary conditions are inadequate and open boundary conditions are required. This technical improvement has been implemented in the work described here.

The rest of the numerical procedure is as discussed in our previous work [20, 21, 22, 23]. For each configuration of color charges sampled for $\Lambda_{s}^{2}$, we solve Hamilton's equations on the lattice for the gauge fields and their conjugate canonical momenta. We compute the space-time evolution of the components of the Stress-Energy tensor, in particular, the two transverse components of the pressure $T^{x x}$ and $T^{y y}$ as well as the energy density $T^{00}$.

In order to calculate $v_{2}$ within our model, we apply the cooling method which was proposed in our previous work [22]. There we obtained, for the total number of classically produced gluons, the equation $N=\sqrt{\frac{8}{\pi}} \int_{0}^{\infty} \frac{d t}{\sqrt{t}} V(t)$ where $V(t)$ is the potential energy for a system of free harmonic oscillators as a function of the cooling time $t$. It is clear that the gluon number defined in this manner is gauge invariant. For $v_{2}$, one can similarly prove that

$$
v_{2}=\frac{\int_{0}^{\infty} \frac{d t}{\sqrt{t}}\left(T^{x x}(t)-T^{y y}(t)\right)}{\int_{0}^{\infty} \frac{d t}{\sqrt{t}} V(t)} .
$$

As in the case of the gluon number, this expression is gauge invariant.

We now turn to our results [25]. In Fig. 3, we plot $\tau T_{x x}$, $\tau T_{y y}$ and the proper time $\tau$ times the energy density $\tau \varepsilon$, in dimensionless units as a function of $\tau$, also in dimensionless units, for a particular value of $\Lambda_{s 0} R$ and impact parameter $b$. We observe that $\tau T_{x x}$ and $\tau T_{y y}$ increase very rapidly at very early times and then decreases quickly as well, before saturating at a much later time. Their magnitudes are the same right after the collision but begin to differ shortly thereafter generating an anisotropy. Note that $\tau \varepsilon$ too rises nearly as rapidly but has a smaller maximum value before relaxing to its asymptotic value. The asymptotic values of 
$\tau T_{x x}$ and $\tau T_{y y}$ differ from each other (and from $\tau \varepsilon$ ). Also, interestingly, the energy density $\varepsilon$ at late times equals the sum of the two components $T_{x x}$ and $T_{y y}$ of the pressure in the transverse plane. All of the described behavior is generic for all values of $\Lambda_{s 0} R$.

Our interpretation of the results presented in Fig. 3 is as follows. The components $T_{x x}$ and $T_{y y}$ of the pressure are spatial gradients of the gauge fields. Even at the earliest times, the gauge fields decrease sharply to the edges of the "almond" characterizing the initial spatial anisotropy. One therefore gets a finite contribution to $T_{x x}$ and $T_{y y}$. Since the initial decrease in the gauge fields at the edges is similar in the $x$ and $y$ directions, the values of $T_{x x}$ and $T_{y y}$ should be similar in magnitude; indeed, Fig. 3 demonstrates that this is the case. Subsequently, the strong non-linear interactions of the gauge fields smooth out their spatial dependence. Eventually, the interactions die off and the system free streams in the transverse plane. This is confirmed by the fact that $\varepsilon \sim T_{x x}+T_{y y}$ at late times.

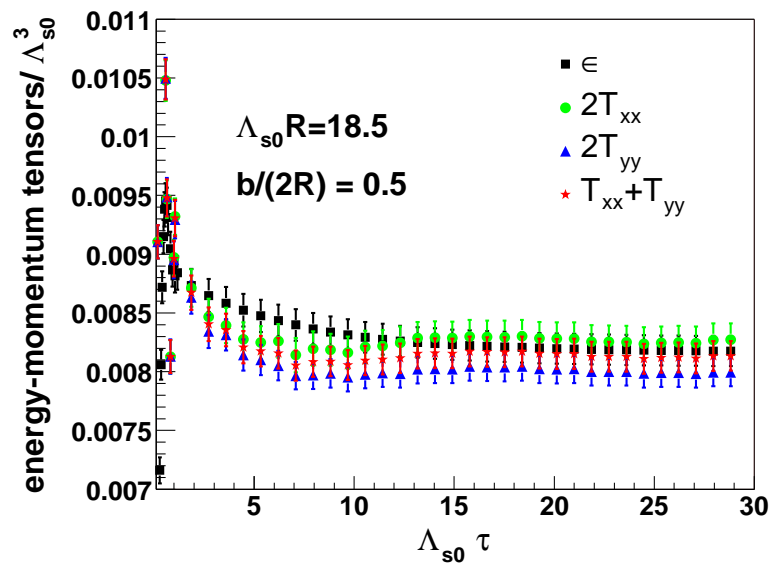

Figure 3. Time evolutions of energy-momentum tensor for $\Lambda_{s} R=$ 18.5 with the impact parameter $b / 2 R=0.6$ Lattice size $128 \times 128$ and $R=32$ are used in the calculation.

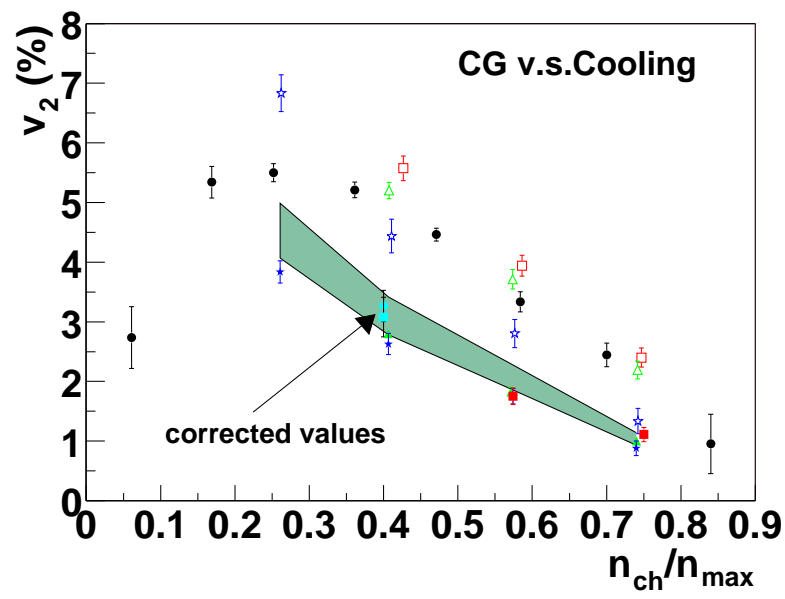

Figure 4. Centrality dependence of $v_{2}$ using cooling (open symbols) and CG (filled symbols). Results are for $\Lambda_{s 0} R=18.5$ (squares), 37 (triangles), and 74 (stars). Full circles denote preliminary STAR data. The band denotes estimated value of $v_{2}$ at very late times. "Corrected values" is the late time cooling and CG result for $\Lambda_{s 0} R=18.5$ at one centrality value.
We now turn to our result for the impact parameter dependence of $v_{2}$. In Fig. 4, we plot $v_{2}$ (computed using the definition in Eq. (7)) versus $n_{c h} / n_{\max }$.

We note, as anticipated, that $v_{2}$ increases with increasing impact parameter. The ratio $n_{c h} / n_{\max }$ is computed selfconsistently within the model. For very peripheral collisions, we expect that the predictions of the model are unreliable since a hard sphere nuclear matter distribution should be replaced by a Wood-Saxon distribution in this regime. The absolute prediction of the model with the data gives about half of the observed $v_{2}$. The rest of the anisotropy must be generated at later times- presumably by hydrodynamic flow. Interestingly, the dependence of $v_{2}$ on $\Lambda_{s 0} R$ is rather weak. For a fixed impact parameter, a prediction of the model is that as $\Lambda_{s 0} R \rightarrow \infty$, we would have the classical contribution to the elliptic flow go to zero: $v_{2} \rightarrow 0$. This is because increasing $\Lambda_{s} R$ is equivalent to increasing $R$ and therefore reducing the initial anisotropy. This again contradicts the trend in the RHIC data suggesting that the late time dynamics is important for the elliptic flow. The momentum distribution $v_{2}\left(p_{t}\right)$ has also been computed in Ref. [25]. The shape and the magnitude of this distribution also disagrees with the RHIC data.

\section{The CGC and RHIC data}

The classical formalism discussed here is applicable only in the initial instants of a nuclear collision. It is inapplicable once the occupation number $f<<1$. Moreover, the final states observed are hadrons while the CGC predicts only the initial distribution of gluons. Subsequent interactions may lead to a thermalized Quark Gluon Plasma. The possibility that the CGC thermalizes has been discussed extensively [33]. It was argued recently that for asymptotic values of the saturation scale $\left(\Lambda_{s} \rightarrow \infty\right)$ the CGC matter does indeed thermalize [34]. For realistic values of the saturation scale, the situation is unclear.

We will assume here, minimally, that gluonic matter formed from the CGC interacts very strongly in the transverse plane at early times and then free streams. Since the typical momentum of the gluons $\left(\sim \Lambda_{s}\right)$ is large than the hadronization scale $\left(\sim \Lambda_{Q C D}\right)$, the gluons may further fragment independently before hadronizing. Invoking partonhadron duality at hadronization then enables us to compare our results to the data.

From the numerical simulations described previously we find that if we fit the total hadron multiplicity at $\sqrt{s_{N N}}=$ $130 \mathrm{GeV}$ (directly equating initial num. of gluons=final num. of hadrons) we find that we obtain a value of $E_{t} / N$ that's proportional to $\Lambda_{s}$ and significantly larger than the observed value (see Tables $1 \& 2$ ). Within the framework of the model, it is clear why this is the case-the CGC overestimates the contributions from high $p_{t}>\Lambda_{s}$-this is more pronounced in $E_{t}$ since it is a more ultraviolet sensitive quantity. A more careful treatment of this regime will reduce the 
global ratio of $E_{t} / N$. One still expect that $E_{t} / N$ to be significantly larger than the measured number. Now $E_{t} / N$ is not a conserved quantity and will reduce due to both independent fragmentation of the gluon "mini-jets" or hydrodynamic flow or both. Which of these is correct will become clearer once more RHIC data is available.

Kharzeev and Nardi [35] have shown that saturation+parton-hadron duality reproduces the centrality dependence of the RHIC data. Further, Kharzeev and Levin [36] have shown that the rapidity and energy dependence of the RHIC data (going from $\sqrt{s_{N N}}=130 \mathrm{GeV}$ to $\sqrt{s_{N} N}=200 \mathrm{GeV}$ ) is predicted accurately in the same scenario. Schaffner-Bielich et al. [37] have shown that the RHIC $p_{t}$ data in a large kinematic region show an $m_{t}$ scaling consistent with saturation. (The saturation scale extracted from the $m_{t}$ scaling of the $p_{t}$ spectra at different centralities reproduces the centrality dependence of the RHIC data.) The flaw in the ointment is the $v_{2}$ data discussed here which disagrees with the RHIC data-this suggests the importance of final state processes and the possible thermalization of produced matter.

On a theoretical level, several improvements can be made to the picture presented here. Firstly, it would be interesting to study the effect of rapidity dependence on the gauge fields -is the system stable under rapidity dependent perturbations? Another interesting, if much more difficult, problem is to match the classical field simulations to a kinetic approach when the occupation numbers fall below unity. This would be the appropriate time at which parton cascade type simulations would be relevant [38]. Finally, how does one extend the renormalization group treatment of the single nucleus problem to that of two nuclei. Despite the formidable challenges, we believe that the QCD based classical formalism presented here is a concrete step towards a theory of high energy heavy ion collisions.

\section{References}

[1] Proceedings of International Symposium on "Statistical Mechanics of Quarks and Hadrons", H. Satz (ed.), Bielefeld, Aug. 24th-31st, 1980, North-Holland Publishers.

[2] J. D. Bjorken, Lectures at Int. Summer Instt. in Theoretical Physics, Current Induced Reactions, Hamburg, Germany, Sept. 15th-26th, 1975, SLAC-PUB 1756.

[3] R. Anishetty, P. Koehler and L. McLerran, Phys. Rev. D22, 2793 (1980).

[4] J. D. Bjorken, Phys. Rev. D27, 140 (1983).

[5] H. Ehtamo, J. Lindfors, and L. McLerran, Z. Phys. C18, 341 (1983).

[6] J. D. Bjorken, J. Kogut, and D. Soper, Phys. Rev. D3, 1382 (1971).

[7] E. A. Kuraev, L. N. Lipatov, V. S. Fadin, Sov. Phys. JETP 45 104 (1977); I. Balitsky and L. N. Lipatov, Sov. J. Nucl. Phys. 28822 (1978).

[8] F. Low, Phys. Rev. D12, 163 (1975); S. Nussinov, Phys. Rev. 14, 246, (1976).
[9] A. Donnachie and P. V. Landshoff, Phys. Lett. B296, 227 (1992).

[10] V. S. Fadin and L. N. Lipatov, Phys. Lett. B429, 127 (1998); M. Ciafaloni and G. Camici, Phys. Lett. B430, 349, (1998).

[11] L.V. Gribov, E. M. Levin and M. G. Ryskin, Phys. Repts. 100 (1983) 1; A. H. Mueller and J.-W. Qiu, Nucl. Phys. B268(1986) 427; J. P. Blaizot and A. H. Mueller, Nucl. Phys. B289 (1987) 847.

[12] K. J. Eskola, K. Kajantie, P. V. Ruuskanen, and K. Tuominen, Nucl. Phys. B570, 379 (2000).

[13] L. McLerran and R. Venugopalan, Phys. Rev. D49 2233 (1994); D49 3352 (1994); D50 2225 (1994).

[14] J. Jalilian-Marian, A. Kovner, L. McLerran and H. Weigert, Phys. Rev. D55 5414 (1997); Y. V. Kovchegov, Phys. Rev. D 54, 5463 (1996).

[15] J. Jalilian-Marian, A. Kovner, A. Leonidov, and H. Weigert, Nucl. Phys. B504 415 (1997); J. Jalilian-Marian, A. Kovner, and H. Weigert, Phys. Rev. D59 014015 (1999); L. McLerran and R. Venugopalan, Phys. Rev. D59 094002 (1999); E. Iancu, A. Leonidov and L. McLerran, Nucl. Phys. A692, 583 (2001); Phys. Lett. B 510, 133 (2001); Phys. Lett. B 510, 145 (2001).

[16] R. V. Gavai and R. Venugopalan, Phys. Rev. D54, 5795 (1996).

[17] E. Iancu, A. Leonidov and L. McLerran, arXiv:hep$\mathrm{ph} / 0202270$.

[18] A. Kovner, L. McLerran and H. Weigert, Phys. Rev, D52, 3809 (1995); D52, 6231 (1995).

[19] M. Gyulassy and L. McLerran, Phys. Rev. C56, 2219 (1997).

[20] A. Krasnitz and R. Venugopalan, hep-ph/9706329, hepph/9808332; Nucl. Phys. B557, 237 (1999).

[21] A. Krasnitz and R. Venugopalan, Phys. Rev. Lett. 84, 4309 (2000).

[22] A. Krasnitz and R. Venugopalan, Phys. Rev. Lett. 86, 1717 (2001).

[23] A. Krasnitz, Y. Nara, and R. Venugopalan, Phys. Rev. Lett. 87, 192302 (2001)

[24] A. Krasnitz, Y. Nara and R. Venugopalan, arXiv:hep$\mathrm{ph} / 0209269$.

[25] A. Krasnitz, Y. Nara and R. Venugopalan, arXiv:hep$\mathrm{ph} / 0204361$.

[26] Y. V. Kovchegov and D. H. Rischke, Phys. Rev. C56, 1084 (1997); S. G. Matinyan, B. Müller and D. H. Rischke, Phys. Rev. C56, 2191 (1997); Phys. Rev. C 57, 1927 (1998); Xiaofeng Guo, Phys. Rev. D59, 094017 (1999).

[27] J.-Y. Ollitraut, Phys. Rev. 46, 229 (1992); Phys. Rev. D48, 1131 (1993).

[28] S. Voloshin and Y. Zhang, Z. Phys. C70, 665 (1996); A.M. Poskanzer and S. Voloshin, Phys. Rev. C58, 1671 (1998).

[29] STAR Collaboration, K.H. Ackermann et al., Phys. Rev. Lett. 86, 402 (2001). The experimental data have been obtained from http://www.star.bnl.gov/STAR/.

[30] P.F.Kolb, P. Huovinen, U. Heinz, and H. Heiselberg, Phys. Lett. 500, 232 (2001). 
[31] X. Wang and M. Gyulassy, Phys. Rev. Lett. 68, 1480 (1992); M. Gyulassy, P. Levai and I. Vitev, Phys. Rev. Lett. 85, 5535, (2000); U. A. Wiedemann, Nucl. Phys. A 690, 731 (2001); R. Baier, D. Schiff and B. G. Zakharov, Ann. Rev. Nucl. Part. Sci. 50, 37 (2000).

[32] M. Bleicher and H. Stöcker, hep-ph/0006147.

[33] A. H. Mueller, Nucl. Phys. B572, 227 (2000); A. H. Mueller, Phys. Lett. B475, 220 (2000); J. Bjoraker and R. Venugopalan, Phys. Rev. C63, 024609 (2001); A. Dumitru and M. Gyulassy, Phys. Lett. B494, 215 (2000).

[34] R. Baier, A. H. Mueller, D. Schiff and D. T. Son, Phys. Lett.
B502, 51 (2001).

[35] D. Kharzeev and M. Nardi, Phys. Lett. B507, 121+ (2001).

[36] D. Kharzeev and E. Levin, nucl-th/0108006.

[37] L. McLerran and J. Schaffner-Bielich, Phys. Lett. B 514, 29 (2001); J. Schaffner-Bielich, D. Kharzeev, L. McLerran, and R. Venugopalan, nucl-th/0108048.

[38] S. A. Bass, nucl-th/0104040; D. Molnar and M. Gyulassy, Phys. Rev. C 62, 054907 (2000); Y. Nara, S. E. Vance and P. Csizmadia, nucl-th/0109018. 